\title{
Particle-Liquid Dynamics of Nuclear Deformations. 1
}

\author{
V.M. Strutinsky and A.G. Magner \\ Institute of Nuclear Research, Kiev, USSR \\ M. Brack \\ Institut für Theoretische Physik, Universität Regensburg, \\ Federal Republic of Germany \\ Received March 9, 1983; revised version December 15, 1983
}

\begin{abstract}
Equations of a combined, liquid plus particle model are derived which accounts consistently for the short-range liquid properties of nuclei as well as for the long-range quasi-particle behavior. Macroscopic quantities are defined as statistical averages and their dynamics is determined by an Euler-type equation, which contains a term due to the interaction with the gas component, and also by the continuity equation. For the quasi-particle component the full quantal treatment is kept in the form of the Liouville equation with a time-dependent external potential related to the bulk density. It may include the residual quasi-particle interaction. It is suggested that the solution of the coupled dynamics is facilitated by the explicit use of an effective sharp nuclear surface as a dynamical variable.
\end{abstract}

\section{Introduction}

Heavy atomic nuclei expose a complexity of features which would ordinarily belong to different physical systems. The liquid drop properties of almost incompressible condensed matter co-exist peacefully with the characteristic behaviour of a quantal gas of nearly independent particles. The existence of single particle and coherent phonon excitations as well as pronounced zones - or gross shell structures - in the energy spectra all require that the quasi-particle correlation length must be larger than the nuclear dimensions. The possibility of such a split behaviour was, in principle, explained by Landau's Fermi-liquid theory $[1,2]$ which played an important role in the development of nuclear theory (see, e.g., Ref. 3). By the Landau theory, the excitations in a system of strongly coupled fermions can, indeed, be described as a gas of interacting quasi-particles. These ideas of the Landau theory have also lead to the formulation of the so-called shell-correction method $[4,5]$ for solving a number of problems of nuclear statics, particularly, at large nuclear distortions. In the last instance, an attempt was made to avoid solving the still unresolved many-body problem of a finite sys- tem of strongly interacting particles by extracting the quasi-particle effects from the liquid-drop background. As a starting point, one considers quantal equations for a finite system of particles which are independent except for their correlations via the common average selfconsistent potential. Macroscopic quantities are determined by means of averaging over the particle phase space and are then identified with and replaced by the analogous quantities of the phenomenological droplet model, not unlike how the equations for the dynamics of liquids are deduced from the particle master equation (see e.g. [6]). The single-particle effects thus arise as selfconsistent corrections to the phenomenological droplet model. They are due to only those singleparticle states which are close to the Fermi energy, which somehow justifies this approach from the point of view of the Fermi-liquid theory. The shellcorrection calculations do not require the knowledge of the true self-consistent potential: a phenomenological deformed single particle potential may be used for the macroscopic part of the average nuclear potential. Neither is required the knowledge of the 
nucleon-nucleon interaction responsible for the bulk properties. Only the effective interaction amplitude of quasi-particles in the vicinity of the Fermi surface must be determined in order to describe the quasiparticle correlations in the nuclear volume.

It is of interest to explore the possibility of a generalization of the static shell-correction approach to taking into account the dynamics of macroscopic motion and the quasi-particle effects. The present paper contains the derivations along this line. It is assumed that the dynamics of the droplet component can be described by a few average quantities such as the mean density, the average velocity and the energy density. On the other hand, the complete quantal treatment is kept for the quasi-particle component, which is dynamically coupled to the macroscopic quantities. The model seems to be able to combine consistently the macroscopic properties of heavy nuclei as densely packed nucleonic systems with the quasi-particle gas features.

\section{Equations of Motion in the "Particle-Liquid Model"}

As in the shell-correction approach, one starts with a proper selfconsistent single particle equation which in the dynamical case now is the selfconsistent Liouville equation for the single particle density matrix $\rho\left(\mathbf{r}_{1} \mathbf{r}_{2} t\right)$

$i \hbar \frac{\partial}{\partial t} \rho=[h, \rho]$,

where $h$ is the selfconsistent single particle Hamiltonian,

$h=T+V$,

$T$ is the kinetic energy operator and the mean potential $V$ is defined by

$V=\operatorname{tr}(v \rho)$,

$v$ being the nucleon-nucleon interaction. The density matrix is normalized to the total number $A$ of particles,

$\operatorname{tr} \rho=A$.

Consider now the Wigner transform of $\rho$ using $\mathbf{r}$ $=\left(\mathbf{r}_{1}+\mathbf{r}_{2}\right) / 2$ and $\mathbf{s}=\mathbf{r}_{1}-\mathbf{r}_{2}$,

$f(\mathbf{r} \mathbf{p} t)=\int d^{3} \mathbf{s} \rho(\mathbf{r}+\mathbf{s} / 2, \mathbf{r}-\mathbf{s} / 2, t) \exp \left(-\frac{i}{\hbar} \mathbf{p s}\right)$.

The current density

$$
\begin{aligned}
\mathbf{j}(\mathbf{r} t) & =-\frac{i \hbar}{m}\left[\boldsymbol{V}_{s} \rho(\mathbf{r}+\mathbf{s} / 2, \mathbf{r}-\mathbf{s} / 2, t)\right]_{\mathbf{s}=0} \\
& =\frac{1}{(2 \pi \hbar)^{3}} \int d^{3} p \frac{1}{m} \mathbf{p} f(\mathbf{r} \mathbf{p} t)
\end{aligned}
$$

and the local density

$\rho(\mathbf{r} t) \equiv \rho(\mathbf{r} \mathbf{r} t)=\frac{1}{(2 \pi h)^{3}} \int d^{3} p f(\mathbf{r p} t)$

are related through the continuity equation

$$
\frac{\partial}{\partial t} \rho(\mathbf{r} t)+\boldsymbol{\nabla} \cdot \mathbf{j}(\mathbf{r} t)=0
$$

For $f(\mathbf{r} p t)$ one has the equation of motion

$$
\frac{\partial}{\partial t} f+\frac{1}{m} \mathbf{p} \cdot \nabla_{r} f-\frac{2}{\hbar} \sin \left(\frac{\hbar}{2}\left(\nabla_{r}^{V} \nabla_{p}^{f}-\nabla_{p}^{V} \nabla_{r}^{f}\right)\right) V^{w} f=0,
$$

where $V^{w}$ is the Wigner transform of $V\left(\mathbf{r}_{1} \mathbf{r}_{2} t\right)$, from which equations for moments of $f$ can be obtained $[7,8]$. Integration of (9) over the p-variable gives the continuity equation (8).

Macroscopic quantities are now introduced as related to a certain component $(\tilde{f})$ of $f$ which, formally, can be determined by averaging in the particle phase space [6]. Performing such an averaging in nuclei might require unrealistically large intervals. It is hoped, however, that this deficiency is not very significant because the averaging does not enter explicity. Moreover, this deficiency is compensated for in the final result by use of simple phenomenological quantities fitted to the observed properties of nuclei. So, neither the realistic distribution function nor its macroscopic part will be required in our model. It will, however, be assumed that the deviation $\left(f_{1}\right)$ of $f$ from the macroscopic distribution $\tilde{f}$ is due to the long-range single-particle effects including those which represent characteristic finite-size features such as the shell structure in the single-particle spectra. We write, correspondingly,

$f=\tilde{f}(\mathbf{r} \mathbf{p} t)+f_{1}(\mathbf{r} \mathbf{p} t)$

and shall try to develop a model which consistently accounts for the interplay between the two components.

The distribution function $\tilde{f}$ determines macroscopic quantities such as the average density

$\tilde{\rho}(\mathbf{r} t)=\frac{1}{(2 \pi \hbar)^{3}} \int d^{3} p \tilde{f}(\mathbf{r} \mathbf{p} t)$, 
the average current density

$\tilde{\mathbf{j}}(\mathbf{r} t)=\frac{1}{(2 \pi \hbar)^{3}} \int d^{3} p \frac{1}{m} \mathbf{p} \tilde{f}(\mathbf{r} \mathbf{p} t)$,

and the average velocity in a given volume element

$\mathbf{u}=\tilde{\mathbf{j}} / \tilde{\rho}$.

The original density may be written as

$\rho(\mathbf{r} t)=\tilde{\rho}(\mathbf{r} t)+\rho_{1}(\mathbf{r} t)$

and similarly the current density

$\mathbf{j}=\tilde{\mathbf{j}}+\mathbf{j}_{1}$.

Equation (8) leads to the macroscopic continuity equation

$\frac{\partial}{\partial t} \tilde{\rho}(\mathbf{r} t)=-\boldsymbol{\nabla} \cdot \tilde{\mathbf{j}}$

which can also be written by means of (13) as

$\frac{\partial}{\partial t} \tilde{\rho}(\mathbf{r} t)=-\boldsymbol{\nabla}(\tilde{\rho} \mathbf{u})$

Equations (16) and (8) require that quantities $\rho_{1}$ and $\mathbf{j}_{1}$ should relate as

$\frac{\partial}{\partial t} \rho_{1}(\mathbf{r} t)+\nabla \cdot \mathbf{j}_{1}(\mathbf{r} t)=0$.

The inverse Wigner transform of $\tilde{f}$ defines some auxiliary density matrix

$\tilde{\rho}\left(\mathbf{r}_{1} \mathbf{r}_{2} t\right)=\frac{1}{(2 \pi \hbar)^{3}} \int d^{3} p \tilde{f}(\mathbf{r} \mathbf{p} t) \exp \left(\frac{i}{\hbar} \mathbf{p} \cdot \mathbf{s}\right)$

which has no immediate physical significance but can be conveniently used in formal derivations. The corresponding inverse Wigner-transform of (10) is

$\rho\left(\mathbf{r}_{1} \mathbf{r}_{2} t\right)=\tilde{\rho}\left(\mathbf{r}_{1} \mathbf{r}_{2} t\right)+\rho_{1}\left(\mathbf{r}_{1} \mathbf{r}_{2} t\right)$,

where $\tilde{\rho}\left(\mathbf{r}_{1} \mathbf{r}_{2} t\right)$ is defined by (19). The strict definition of $\tilde{f}$ as an average of $f$ would require that the local average of the density $\rho_{1}$ should vanish,

$\left\langle\rho_{1}(\mathbf{r} t)\right\rangle_{\mathbf{A v}}=0$.

This property will not be required in the following derivations. It must be assumed, however, that

$\operatorname{tr} \rho_{1}=0$,

as it follows from (4) and (20). Substitution of (21) into the original equation (1) gives

$$
\begin{aligned}
& i \hbar \frac{\partial}{\partial t} \tilde{\rho}\left(\mathbf{r}_{1} \mathbf{r}_{2} t\right)-\left[\tilde{h^{t}}, \tilde{\rho}\right]-\left[V_{1}, \tilde{\rho}\right] \\
& =-i \hbar \frac{\partial}{\partial t} \rho_{1}\left(\mathbf{r}_{1} \mathbf{r}_{2} t\right)+\left[\tilde{h}^{t}, \rho_{1}\right]+\left[V_{1}, \rho_{1}\right] .
\end{aligned}
$$

Here,

$\tilde{h^{t}}=T+\tilde{V}$,

where

$\tilde{V}\left(\mathbf{r}_{1} t\right)=\operatorname{tr}_{2}\left(v\left(\mathbf{r}_{1} \mathbf{r}_{2}\right) \tilde{\rho}\left(\mathbf{r}_{2} t\right)\right)$

and

$V_{1}\left(\mathbf{r}_{1} t\right)=\operatorname{tr}_{2}\left(v\left(\mathbf{r}_{1} \mathbf{r}_{2}\right) \tilde{\rho}\left(\mathbf{r}_{2} t\right)\right)$.

The above equations have been derived on a purely formal level. In the following, we shall try to interpret physically the quantities introduced above.

We now assume that $f$ corresponds to a locally quasistatic equilibrium of the nuclear matter distribution and take it in the form

$\tilde{f}(\mathbf{r} \mathbf{p} t)=F(\mathbf{r q} t), \quad \mathbf{q}=\mathbf{p}-m \mathbf{u}$,

where $F$ is a function of $\mathbf{q}^{2}$ only. For real dense matter this is a rather natural assumption, provided that the macroscopic fluid velocity $\mathbf{u}$ is small as compared to the characteristic nucleon velocity which correspond to energies of the order of several tens of $\mathrm{MeV}$. By performing the Wigner-transformation and then taking the first p-moment of both sides of (23) one obtains

$$
\begin{aligned}
& \frac{\partial}{\partial t} \tilde{\mathbf{j}}_{v}+\sum_{\mu} \frac{\partial}{\partial r_{\mu}}\left(\tilde{\rho} u_{v} u_{\mu}\right) \\
& \quad+\frac{1}{m} \sum_{\mu} \frac{\partial}{\partial r_{\mu}} P_{v \mu}+\frac{1}{m} \tilde{\rho} \frac{\partial}{\partial r_{v}}\left(\tilde{\boldsymbol{V}}+V_{1}\right) \\
& =\frac{1}{i \hbar(2 \pi \hbar)^{3}} \int d^{3} p \frac{1}{m} p_{v} \int d^{3} s\left(-i \hbar \frac{\partial}{\partial t} \rho_{1}\right. \\
& \left.\quad+\left[\tilde{h}^{t}, \rho_{1}\right]+\left[V_{1}, \rho_{1}\right]\right) \exp \left(-\frac{i}{\hbar} \mathbf{p} \cdot \mathbf{s}\right),
\end{aligned}
$$

where

$P_{v \mu}(\mathbf{r} t)=\frac{1}{(2 \pi \hbar)^{3} m} \int d^{3} p\left(p_{v}-m u_{v}\right)\left(p_{\mu}-m u_{\mu}\right)$

$\cdot F(\mathbf{r}, \mathbf{p}-m \mathbf{u}, t)=\frac{2}{3} \delta_{v \mu} \mathscr{E}_{\mathrm{kin}}(\mathbf{r} t)$

is the macroscopic pressure tensor. This quantity will be assumed to be diagonal and independent of u. In (29), $\mathscr{E}_{\text {kin }}$ is the local average density of the kinetic energy of the particles in the local frame moving with the velocity $\mathbf{u}$.

Now, it can be required that $\rho_{1}$ is chosen in such a way that the integrand on the r.h.s. of (28) should 
vanish. This is achieved if $\rho_{1}$ is a solution to the Liouville-type equation,

$i \hbar \frac{\partial}{\partial t} \rho_{1}=\left[\tilde{h}^{t}, \rho_{1}\right]+\left[V_{1}, \rho_{1}\right]$.

The single-particle Hamiltonian $\tilde{h}^{t}$, see (24), contains the time-dependent potential $\tilde{V}$, which is an external quantity with respect to $\rho_{1}$. The second term on the r.h.s. of (30) is the self-consistency correction. It is of second order in $\rho_{1}$ and without it (30) is just the equation of the cranking model, also familiar from other theories dealing with time-dependent constraints. However, the solution $\rho_{1}$ to (30) which we consider here is different in that respect that it is normalized to zero according to (22). Note, that such a renormalization corresponds closely to the particle-hole normalization in Landau's Fermi liquid theory [1], Chap. 1. The quantity $f_{1}$ in (10) is related to $\rho_{1}$ as

$f_{1}(\mathbf{r} \mathbf{p} t)=\int d^{3} s \rho_{1}(\mathbf{r}+\mathbf{s} / 2, \mathbf{r}-\mathbf{s} / 2, t) \exp \left(-\frac{i}{\hbar} \mathbf{p} \cdot \mathbf{s}\right)$.

With the density matrix $\rho_{1}$ which satisfies (30) the continuity equation (18) turns into identity. The reason for our definition of $\rho_{1}$ becomes clear. It is hoped that one or another nuclear theory can be used in obtaining specific solutions to (30). This can, for example; be the adiabatic approximation or the RPA-theory. (In the latter case, the interaction term containing $V_{1}$ must be included). A solution can be found also in the semiclassical case when (30) can be reduced in the nuclear interiour to Landau's equations for the distribution function of zerosound. Assuming that (30) is fulfilled, one can transform (28) to the form

$\frac{\partial}{\partial t}\left(\tilde{\rho} u_{v}\right)+\sum_{\mu} \frac{\partial}{\partial r_{\mu}}\left(\tilde{\rho} u_{v} u_{\mu}\right)+\frac{1}{m} \tilde{\rho} \frac{\partial}{\partial r_{v}}\left(\frac{\delta \mathscr{E}}{\delta \tilde{\rho}}+V_{1}\right)=0$

In the derivation of this equation we replaced the sum of $\boldsymbol{\nabla} P / \tilde{\rho}$ and $\boldsymbol{V} \tilde{V}$ by the gradient of the functional derivative of the local average macroscopic energy density $\mathscr{E}$ with respect to $\tilde{\rho}$ thus assuming $\mathscr{E}$ to be a functional of the local density $\tilde{\rho}$ (see, e.g. [911]). Making use of the macroscopic continuity equation (17), equation (32) can be transformed into the standard form of the hydrodynamical Euler equation [11]

$\frac{D \mathbf{u}}{D t}=-\frac{1}{m} \nabla\left(\frac{\delta \mathscr{E}}{\delta \tilde{\rho}}+V_{1}\right)$

where

$\frac{D \mathbf{u}}{D t}=\frac{\partial}{\partial t} \mathbf{u}+(\mathbf{u} \cdot \boldsymbol{V}) \mathbf{u}$ is the macroscopic acceleration of a mass element. It should be noted that (33) contains the correction $V_{1}$ due to the coupling to the quasi-particle modes. Below it is indicated how such equations of coupled dynamics can be resolved. As it is clear from the above definitions, the particular form of the coupling term in (34) is closely related to our choice of $\rho_{1}$ as a solution to (33) and it would take on a different form should another definition of $\rho_{1}$ be accepted.

It can be noted that the hydrodynamical form of (33) is due to our assumption in (27) of a localequilibirium form for $\tilde{f}$. Our model can be modified without difficulty if approximation (27) turns out to be insufficient for the macroscopic component. Also the viscosity due to the short-range particle interaction can be included as a phenomenological quantity.

\section{Conservation of the Total Energy}

Together with (17) and (30), equation (33) determines the macroscopic dynamics - affected by the quasiparticle correlations - in terms of the time evolution of two macroscopic quantities, namely, the average density $\tilde{\rho}(\mathbf{r} t)$ and the average velocity $\mathbf{u}(\mathbf{r} t)$. It will now be shown that the total energy of the system is conserved. The energy includes the quasi-particle energy correction as well as the macroscopic energy defined in the usual manner. In order to prove this, we multiply (32) by $\mathbf{j}(\mathbf{r} t)$ and integrate over the spatial coordinates. Using once more the continuity equation we get after integration,

$\frac{d}{d t} \tilde{E}+\int d^{3} r V_{1}(\mathbf{r} t) \frac{\partial}{\partial t} \tilde{\rho}(\mathbf{r} t)$,

where

$\tilde{E}=\int d^{3} r\left(\frac{m}{2} \tilde{\rho} \mathbf{u}^{2}+\mathscr{E}(\tilde{\rho})\right)$

is the macroscopic energy which includes a collective kinetic part. The second term in $(35)$ is the time derivative of the quantal single-particle energy correction $E_{1}$ which is a functional of $\tilde{\rho}(t)$,

$E_{1}=\operatorname{tr}\left(\tilde{h}^{t} \rho_{1}\right)+\frac{1}{2} \operatorname{tr}\left(V_{1} \rho_{1}\right)$.

Indeed, from this definition and from (26) one obtains

$$
\begin{aligned}
\frac{d}{d t} E_{1}= & \operatorname{tr}\left(\rho_{1} \frac{\partial}{\partial t} \tilde{h}^{t}\right)+\operatorname{tr}\left(\tilde{h}^{t} \frac{\partial}{\partial t} \rho_{1}\right) \\
& +\operatorname{tr}\left(V_{1} \frac{\partial}{\partial t} \rho_{1}\right) .
\end{aligned}
$$


Neglecting terms of third order in $\rho_{1}$ one has

$\frac{d}{d t} E_{1}=\operatorname{tr}\left(\rho_{1} \frac{\partial}{\partial t} \tilde{V}\right)$.

This is so because, according to (30), the two last terms in (38) cancel each other. Taking into account (25) and (26) it can also be written as

$\frac{d}{d t} E_{1}=\int d^{3} r \cdot V_{1} \frac{\partial}{\partial t} \tilde{\rho}$

Thus, the conserved total energy is the sum

$E=\tilde{E}+E_{1}$,

although each of its two components separately is a time dependent quantity.

It may be illustrative to derive the total energy expression (41) directly from its microscopic definition as

$E=\operatorname{tr}(T \rho)+\frac{1}{2} \operatorname{tr}(V \rho)$.

It can be written as

$\operatorname{tr}(T \tilde{\rho})+\frac{1}{2} \operatorname{tr}(\tilde{V} \tilde{\rho})+\operatorname{tr}\left(\tilde{h}^{t} \rho_{1}\right)+\frac{1}{2} \operatorname{tr}\left(V_{1} \rho_{1}\right)$.

The two last terms here are just the $E_{1}$-component (37). Consider now the time derivative of the first two terms in (43). Taking into account the definition (25) of $\tilde{V}$, we write

$$
\begin{aligned}
& \frac{d}{d t} \operatorname{tr}\left(T \tilde{\rho}+\frac{1}{2} \tilde{V} \tilde{\rho}\right)=\operatorname{tr}\left(\tilde{h}^{t} \frac{\partial}{\partial t} \tilde{\rho}\right) \\
& =\int d^{3} r_{1} \int d^{3} r_{2} \delta\left(\mathbf{r}_{1}-\mathbf{r}_{2}\right) \\
& \quad \cdot\left(-\frac{h^{2}}{2 m} \Delta_{r_{1}}+\tilde{V}\left(\mathbf{r}_{1} t\right)\right) \frac{\partial}{\partial t} \tilde{\rho}\left(\mathbf{r}_{1} \mathbf{r}_{2} t\right) .
\end{aligned}
$$

In terms of $(\mathbf{r}, \mathbf{s})$-variables

$$
\Delta_{r_{1}}=\frac{1}{4} \Delta_{r}+\nabla_{r} \cdot \nabla_{s}+\Delta_{s}
$$

and using (19) we rewrite the r.h.s. of (44) as

$$
\begin{aligned}
& \frac{1}{(2 \pi \hbar)^{3}} \int d^{3} r \int d^{3} s \int d^{3} p \delta(\mathbf{s}) \\
& \cdot\left(-\frac{\hbar^{2}}{2 m} \Delta_{s}+\tilde{V}(\mathbf{r}+\mathbf{s} / 2, t)\right) \frac{\partial}{\partial t} \tilde{f} \exp \left(\frac{i}{\hbar} \mathbf{p} \cdot \mathbf{s}\right) .
\end{aligned}
$$

By Gauss' theorem, the first two terms of (45) vanish. The integral (46) is, hence, readily reduced to

$$
\frac{d}{d t} \int d^{3} r\left(\frac{m}{2} \tilde{\rho} u^{2}+\mathscr{E}_{\text {kin }}\right)+\int d^{3} r \tilde{V} \frac{\partial}{\partial t} \tilde{\rho},
$$

where $\mathscr{E}_{\text {kin }}$ is the average local kinetic energy density of the nucleons, see (25). By means of (26), it can be proven that the expression (47) is indentical to $d \tilde{E} / d t$ in (35). So, the microscopic energy (42) is, indeed, equal to the energy defined as in (41) with the accuracy of the second order terms in $\rho_{1}$.

\section{Discussion of the Quasi-Particle Corrections}

We consider now several simple, though not always realistic, examples with the purpose of illustrating the physical significance of the coupling terms. Here, we only deal with the quasi-stationary component of $\rho_{1}$ which is assumed to be uniquely determined by $\tilde{\rho}$ and its time derivatives. More general solutions to (30) may contain dynamic contributions corresponding to different states of the intrinsic excitations, such as, for example, the zero-sound modes. It should be noted, however, that quasi-stationarity has not been assumed in the above derivations and, in particular, in proving the energy conservation.

The physical significance of the correction $V_{1}$ in (33) can be clarified in the following way. First, consider the extreme limit when $\rho_{1}$ is approximated by the solution $\rho_{1}^{(s)}$ of the static equation

$\left[\tilde{h}^{t}, \rho_{1}^{(s)}\right]=0$

for each given $\tilde{\rho}(\mathbf{r} t)$. The solution to (48) is a certain real and symmetric density matrix and the time variable enters there only as a parameter through $\tilde{\rho}$. Such a solution to (48) which corresponds to the lowest single particle energy at each distortion of $\tilde{V}$ and satisfies the constraints (21), (22) is already known. It is the shell-correction density matrix

$$
\begin{aligned}
& \delta \rho_{s}\left(\mathbf{r}_{1} \mathbf{r}_{2}\right) \equiv \delta \rho[\tilde{\rho}(t)] \\
& =\Sigma_{\alpha} \delta n_{\alpha} \varphi_{\alpha}\left(\mathbf{r}_{1}\right) \varphi_{\alpha}^{*}\left(\mathbf{r}_{2}\right) .
\end{aligned}
$$

Here, $\varphi_{\alpha}$ and $e_{\alpha}$ are the eigenfunctions and energies of the deformed single-particle Hamiltonian $\tilde{h}^{t}$. The quantity

$\delta n_{\alpha} \equiv \delta n\left(e_{\alpha}\right)=n_{\alpha}-\tilde{n}_{\alpha}$

is the difference between the occupation number for the $\alpha$-th single-particle state and the corresponding smoothed quantity, see e.g. [5]. The energy $E_{1}$ (37) in this case is the so-called shell-correction energy and the related correction $V_{1}$ in (33) corresponds to adding the shell energy corrections to the macroscopic potential energy of distortion. A relationship equivalent to (39) determines the driving force due to the shell structure; it has been considered in details in [5]. The relationship between the quasiparticle shell effects and the restoring force due to 
the distortion of the Fermi-sphere in the so-called long-range fluid dynamics is discussed in [12].

It is of interest to show that the $V_{1}$-coupling term in (33) may also contain corrections to the inertia of the bulk distribution. The inertial properties are not altered as long as $\delta \rho_{1}$ remains real, as it was in the quasi-static case. However, corrections to the mass coefficients appear already in the simplest dynamical theories, such as e.g., the cranking model. In lowest order perturbation theory one writes

$\rho_{1}=\rho_{1}^{(s)}+\rho_{1}^{(d)}=\delta \rho_{s}+\rho_{1}^{(d)}$,

where $\delta \rho_{s}$ is defined as in (49) and one determines the dynamical part $\rho_{1}^{(d)}$ as a solution to the nonuniform equation

$i \hbar \frac{\partial}{\partial t} \rho_{1}^{(d)}-\left[\tilde{h}^{t}, \rho_{1}^{(d)}\right]=-i \hbar \frac{\partial}{\partial t} \delta \rho_{s}$.

We now assume that the time-dependence of $\tilde{\rho}$ is specified in terms of a "collective variable" $a(t)$ and define the following one-body operator

$\hat{q}=\frac{\partial}{\partial a} \tilde{h}^{t}=\frac{\partial}{\partial a} \tilde{V}$.

According to (39),

$\frac{d}{d t} E_{1}=\dot{a} Q_{1}$,

where $Q_{1}$ is the "deformation moment" defined as

$Q_{1}=\operatorname{tr}\left(\hat{q} \rho_{1}\right)=Q_{1}^{(s)}+Q_{1}^{(d)}$,

in correspondence with (51). The $Q_{1}^{(s)}$-component in (55) adds the static shell energy correction to the deformation potential, as explained above. In firstorder perturbation theory one finds the matrix elements

$$
\begin{aligned}
\left(\rho_{1}^{(d)}\right)_{\alpha \beta}= & \left(\delta n_{\alpha}-\delta n_{\beta}\right) \partial_{\alpha \beta}(a) \\
& \cdot \int_{0}^{t} d t^{\prime} \dot{a}\left(t^{\prime}\right) \exp \left(\frac{1}{i \hbar}\left(e_{\alpha \beta}-i \eta\right)\left(t-t^{\prime}\right)\right),
\end{aligned}
$$

where $\eta \rightarrow+0$

$\partial_{\alpha \beta}(a)=\left\langle\varphi_{\alpha} \mid \frac{\partial}{\partial a} \varphi_{\beta}\right\rangle=q_{\beta \alpha} / e_{\beta \alpha}$

and

$e_{\beta \alpha}=e_{\beta}-e_{\alpha}$.

The quantity $Q_{1}^{(d)}$ is given by

$$
\begin{aligned}
Q_{1}^{(d)}= & \Sigma_{\alpha \beta}\left(\rho_{1}^{(d)}\right)_{\alpha \beta} q_{\beta \alpha} \\
= & \Sigma_{\alpha \beta}\left(\delta n_{\alpha}-\delta n_{\beta}\right) e_{\beta \alpha} \partial_{\beta \alpha}^{2} \\
& \cdot \int_{0}^{t} d t^{\prime} \cos \left(\frac{1}{\hbar}\left(t-t^{\prime}\right) e_{\beta \alpha}\right) \exp \left(-\frac{\eta}{\hbar}\left(t-t^{\prime}\right)\right) .
\end{aligned}
$$

For a smooth $a(t)$ we can write

$\dot{a}\left(t^{\prime}\right)=\dot{a}(t)-\left(t-t^{\prime}\right) \ddot{a}(t)$.

When substituted in (58) and (54), the first term in (59) would produce in $d E_{1} / d t$ a term proportional to $\dot{a}^{2}$ which disappears within our approximation. The second term in (59) contributes to the time rate of change of the kinetic energy

$\frac{d}{d t} E_{1}^{(\mathrm{kin})}=B_{1} \dot{a} \ddot{a}$.

The mass coefficient $B_{1}$ is

$B_{1}=\hbar^{2} \Sigma_{\alpha \beta}\left(\delta n_{\alpha}-\delta n_{\beta}\right) \partial_{\alpha \beta}^{2} / e_{\beta \alpha}$.

Note that the diagonal matrix elements $\left(\rho_{1}^{(a)}\right)_{\alpha \alpha}$ are of second order in the small quantities and, hence, they are proportional to $\dot{a}^{2}$. Therefore, condition (22) is not violated in this approximation.

The cranking-type mass parameter $B_{1}$ should be considered as a particle correction to the macroscopic value contained in the first part of (36) if the collective variable $a(t)$ determines also the dynamics of $\tilde{\rho}(t)$. The expression (61) resembles the familiar one in the ordinary cranking model calculations. The difference is that $\delta n_{\alpha}$ in (61) replaces the ordinary occupation numbers. However, it leads to important changes because in (61) contributions from matrix elements between states more distant from the Fermi energy than the gross shell spacing $\hbar \Omega$ are effectively cut off. Such transitions are just the ones known to be responsible for the so-called hydrodynamical component of the mass parameter. Apparently, this makes the dual model suggested here more consistent. The derivation of $B_{1}$ was presented here for a purely illustrative purpose because any realistic calculation of intertial parameters for large amplitude distortions and finite collective velocities requires a significantly more thorough analysis.

\section{Conclusion}

The dual, $\mathrm{Li}-\mathrm{Pa}$ model approach suggested in this paper can be considered as an alternative to the catholic advent of the TDHF theories and it can be of particular interest if it turns true - as some recent experiments also show [13] - that the gas properties 
of nuclei are not as overwhelming as currently believed. The impact of the Hartree-Fock calculations was due to the use of effective Skyrme forces fitted to the static properties of nuclei, and in many respects the Hartree-Fock model corresponds to the orthodox droplet model. In the static case, the constrained Hartree-Fock (CHF) calculations finally proved to be a not very practical alternative to the combined liquid-drop + gas shell correction approximation. It is hoped that the $\mathrm{Li}-\mathrm{Pa}$ model can play an analogous role in the dynamical theory.

The actual solution of the coupled dynamical equations can be eased because the coupling is weak within the nuclear volume where the gradient of the average nuclear potential $V$ is relatively small. This makes it possible to reduce the equations in the nuclear volume to simpler linearized forms analogous to those considered in the theories of infinite matter. (This statement does not concern the shell structure which is due to repeated reflections from edges of the potential well and the formation of closed periodic classical paths). An important example is our equation (30) for the $\rho_{1}$-component, which in the semi-classical limit can be reduced to the Landau equation for the particle-hole distribution function relevant for zero-sound excitation [14].

Stronger coupling takes place near the edge of the nucleus where the density gradient is large. In this region special approximations can be developed which explicity employ as a time-dependent dynamical variable the concept of a sharp effective surface of the density distribution. Considering the $\mathrm{Li}-\mathrm{Pa}$ equations in this specific region, certain simple and, in fact, rather well known boundary conditions for the internal region can be obtained which determine the physical solution. These boundary conditions establish the coupling between the particle dynamics inside the nuclear volume and the dynamics of the nuclear shape $[15]$. In $[16,17]$ this method has successfully been applied to nuclear giant resonances of the isoscalar type. It should also be noted that the problem of giant nuclear resonances was approached by several authors by employing certain ad hoc boundary conditions [18-20]. As a consequence, unsatisfactory results were obtained. In contrast, in this combined model all necessary quantities appear naturally and the boundary conditions are directly obtained from the $\mathrm{Li}-\mathrm{Pa}$ equations. In addition, the validity of our approach is not necessarily restricted to small amplitude processes.
The authors express their gratitude to Dr. R. Hasse for many stimulating discussions and help in preparing the manuscript. One of the authors (M.B.) is grateful to the Institute of Nuclear Research and the Academy of Science of the Ukrainian SSR for the hospitality extended to him during a visit. He also gratefully acknowledges a travel grant of the Deutsche Forschungsgemeinschaft.

\section{References}

1. Lifshitz, E.M., Pitacvsky, L.P.: Statistical physics. Part 2. Moscow: Nauka 1978

2. Abrikosov, A.A., Gorkov, L.P., Dzjaloshinsky, I.E.: Methods of quantum field theory in statistical physics. Moscow: Fizmatgiz 1962

3. Migdal, A.B.: Theory of finite fermi liquids and properties of atomic nuclei. Moscow: Nauka 1965

4. Strutinsky, V.M.: Nucl. Phys. A 95, 420 (1967); A 122, 1 (1968)

5. Brack, M., Damgaard, V., Jensen, A., Pauli, H.C., Strutinsky, V.M., Wong, C.Y.: Rev. Mod. Phys. 44, 320 (1972)

6. Lifshitz, E.M., Pitaevsky, L.P.: Physical kinetics. Moscow: Nauka 1979

7. Gronewald, H.J.: Physica, 12, 405 (1946)

8. Moyel, J.E.: Proc. Cambridge Philos. Soc. 45, 99 (1949)

9. Holzwarth, G., Eckart, G.: Nucl. Phys. A 325, 1 (1979)

10. Brack, M., Guet, C., Hakansson, H.-B., Magner, A., Strutinsky, V.M.: 4th Int. Conf. on Nuclei far from Stability, Helsingør, p. 65. Geneva: CERN 81-09 1981

11. Landar, L.D., Lifshitz, E.M.: Fluid mechanics. London: Pergamon Press 1959

12. Brack, M.: Phys. Lett. B 123 B, 143 (1983)

13. Nix, J.R.: Comments on nuclear and particle physics. 1983; Preprint LA-UR-82-3651

14. Magner, A.G., Strutinsky, V.M.: (to be published)

15. Strutinsky, V.M., Magner, A.G., Brack, M.: (to be published)

16. Strutinsky, V.M., Magner, A.G., Denisov, V.Yu.: Z. Phys. A Atoms and Nuclei 315, 301 (1984)

17. Denisov, V.Yu., Magner, A.G.: (to be published)

18. Akhiezer, I.A., Barz, B.I., Lazurin-Elzufin, V.G.: Yad. Fiz. (USSR) 15, 863 (1972)

19. Eckart, G., Holzwarth, G., Da Providencia, J.P.: Nucl. Phys. A 364, 1 (1981)

20. Hasse, R.W., Ghosh, G., Winter, V.M., Lambroso, A.: Phys. Rev. 25 C, 2771 (1982)

V.M. Strutinsky

A.G. Magner

Institute for Nuclear Research

$252028 \mathrm{Kiev}$, USSR

M. Brack

Institut für Theoretische Physik

Universität Regensburg

D-8400 Regensburg

Federal Republic of Germany 